\title{
Electrospray makes molecular elephants fly
}

The 'electrospray revolution' in the late 1980s dramatically increased the utility of mass spectrometry. Developed by John Fenn at Yale University, electrospray ionization (ESI) finally allowed the mass spectrometric analysis of biological macromolecules, including proteins, and was rapidly adopted in laboratories around the world. But the seeds of the electrospray revolution had been planted long before.

In 1968, Malcolm Dole at Northwestern University found that large gas-phase ions ('macroions') could be transiently generated by "electrospraying a dilute solution into an evaporation chamber containing nitrogen, and by allowing the volatile solvent to evaporate from the tiny drops so produced." Dole generated a spray of charged liquid drops by streaming a dilute polymer solution through a needle held at high voltage relative to the spray chamber wall. Once electrosprayed into the evaporation chamber, polymer ions were produced in the ambient gas by the interplay of evaporation and electrostatic repulsion.

Lord Rayleigh had reported in 1882 that as a solvent evaporates, the charge density on the surface of a "charged spherical mass of liquid" increases until electrical repulsion overcomes surface tension, causing the droplet to disintegrate. In Dole's apparatus, repetitive disintegrations resulted in droplets so small that they contained only a single macroion, whose mass could then be determined.

Unfortunately, the mass spectrometers available at the time could not detect singly charged ions with masses larger than a few thousand daltons, well below the mass range for macromolecules of biological interest. Also, Dole had overlooked the problem of ion re-solvation occurring prior to mass measurement. Owing to the substantial temperature drop during its adiabatic expansion, the electrosprayed aerosol would rapidly become saturated with solvent vapor. Hence, Dole's macroions would get re-solvated, and their measured masses would be higher than the actual values by an unknown amount.

John Fenn soon became interested in Dole's research, but at first he was unable to overcome the problems Dole had encountered. Almost 20 years passed before Fenn resumed working on the electrospray technique, starting with small molecules such as vitamin $B_{6}$ and using a quadrupole mass analyzer (Milestone 6). With a newly designed electrospray apparatus, Fenn and colleagues obtained spectra of a variety of small molecules, and observed "peaks corresponding, for example, to protons solvated by various combinations and numbers of methanol and water molecules that could be varied widely simply by adjusting the flow rate and/or temperature of the counter-current drying gas." More importantly, they were able to generate intact ions of fragile molecules (without fragmentation) for mass spectrometric analysis. Electrospray ionization mass spectrometry (ESI-MS) was born.

Fenn then analyzed larger molecules-such as the antibiotic gramicidin $\mathrm{S}$ and the immunosuppressant cyclosporin A-and observed peaks corresponding to singly and multiply charged ions with intensities that depended on the composition and concentration of the solutions. The observation of multiply charged ions would be the fundamental step toward extending the application of ESI-MS to macromolecules, because the mass range of the mass analyzers could now be increased by a factor equal to the number of charges on a macroion.

By extensively studying polyethylene glycol oligomers, Fenn determined how multiply charged ions are generated and how to control that process. Mass spectrometric analysis of proteins immediately followed. Electrospray ionization is currently the technique typically used to generate ions in liquid chromatographymass spectrometry (Milestone 8) and is broadly used in many biological and biomedical applications, including proteomics

(Milestone 20) and drug-discovery efforts.

In 2002, John Fenn was awarded the Nobel Prize in Chemistry together with Koichi Tanaka for the "development of soft desorption ionisation methods for mass spectrometric analyses of biological macromolecules" (see Milestone 18). As he quipped in his Nobel Prize lecture, Fenn became a Nobel laureate for giving "electrospray wings to molecular elephants."

Cosma D Dellisanti, Associate Editor, Nature Structural \& Molecular Biology

PRIMARY RESEARCH PAPERS Dole, M. et al. Molecular beams
of macroions. J. Chem. Phys. 49, 2240-2249 (1968) | Yamashita,
M. \& Fenn, J.B. Electrospray ion source. Another variation on the
free-jet theme. J. Phys. Chem. 88, 4451-4459 (1984) |
Whitehouse, C.M., Dreyer, R.N., Yamashita, M. \& Fenn, J.B.
Electrospray interface for liquid chromatographs and mass
spectrometers. Anal. Chem. 57, 675-679 (1985) | Fenn, J.B.,
Mann, M., Meng, C.K., Wong, S.F. \& Whitehouse, C.M.
Electrospray ionization for mass spectrometry of large
biomolecules. Science 246, 64-71 (1989)
FURTHER READING Rayleigh, J.S.W. On the equilibrium of liquid
conducting masses charged with electricity. Philos. Mag. 14, 184-
186 (1882) | Wong, S.F., Meng, C.K. \& Fenn, J.B. Multiple charging
in electrospray ionization of poly (ethylene glycols). J. Phys. Chem.
92, 546-550 (1988) | Fenn, J.B. Electrospray ionization mass
spectrometry: how it all began. J. Biomol. Tech. 13, 101-118
(2002) | Fenn, J.B. Nobel Lecture: Electrospray wings for
molecular elephants (Nobel Foundation, 2002)

